Methods: Thirty-two SSc patients underwent a cardiac MRI with dedicated lung scanning and chest HRCT on the same day. One-hundred-thirty-five regions of interest (ROIs) were identified, and STIR and T1 sequences were acquired[before (T0) and after 5 (T5), 10 (T10) and 15 (T15) minutes from gadolinium injection]. The ROls were classified according to HRCT as normal, dependent areas (probably related to blood pooling in supine position) and pathological areas(ground glass \pm reticulation on HRCT). Mean STIR and T1 times were also calculated for each patients, and correlated with FVC, DLco, B-lines on lung ultrasound and HRCT semi-quantitative scoring (Scleroderma Lung Study score). Patients were followed-up and lung worsening was defined on the basis of clinical judgement and at least $>15 \%$ DLco decline.

Results: Mean STIR and mean T1 times were significantly different between normal, dependent and pathologic areas ( $p<0.001$ between groups). Patients' mean STIR showed a significant correlation with DLco $(R=-0.56, p<0.01)$, HRCT Scleroderma Lung Study score $(R=0.52, p<0.01)$ and $B$-lines on lung ultrasound $(R=0.63, p<0.01)$. The mean STIR of the 10 patients who developed a worsening pulmonary involvement had significantly different MRI signal intensity in comparison to the 25 patients without worsening pulmonary involvement $(125 \pm 46$ vs 66 \pm 37 msec, $p<0.01$ ).

Conclusions: Our data highlight the usefulness of lung MRI in SSc patients to differentiate normal, dependent and pathologic areas, without need for contrast medium administration, and with good correspondence to other functional and imaging parameters. STIR values may have prognostic implications to predict lung worsening. Lung MRI, although still very preliminary, is a promising imaging tool that in the future may integrate HRCT in SSc-related ILD.

Disclosure of Interest: None declared

DOI: 10.1136/annrheumdis-2018-eular.6840

\section{FRI0440 $\quad$ RENAL INVOLVEMENT IN MIXED CONNECTIVE TISSUE DISEASE: A SINGLE CENTREEXPERIENCE}

\section{E. Martin Nares ${ }^{1}$,S.E. Ramírez Andrade ${ }^{2}$, N.O. Uribe Uribe ${ }^{3}$, T.S. Rodríguez} Reyna ${ }^{1} .{ }^{1}$ Immunology and Rheumatology Department, ${ }^{2}$ Nephrology Department; ${ }^{3}$ Pathology Department, Instituto Nacional de Ciencias Médicas y Nutrición Salvador Zubirán, Mexico City, Mexico

Background: Kidney injury in mixed connective tissue disease (MCTD) is an uncommon manifestation. Prevalence has been reported to be $<4 \%$ in some cohorts. The frequency of renal involvement in Hispanic patients with MCTD is not known.

Objectives: We aimed to describe the prevalence, clinical characteristics and outcomes of renal involvement in Mexican patients with MCTD

Methods: We conducted a retrospective single-centre study. We included patients with a diagnosis of MCTD according to the Alarcón-Segovia criteria who regularly attended to a referral centre in Mexico City (2003-2017) and we identified those with renal involvement defined as proteinuria $>500 \mathrm{mg} / \mathrm{d}$ with or without active sediment, creatinine elevation $50 \%$ above baseline or development of glomerular filtration rate (GFR) $<60 \mathrm{ml} / \mathrm{min}$, with no other known cause. We collected demographics, clinical manifestations, follow-up time, treatment, outcomes and damage (SLICC/ACR-DI), renal function, serological and histological variables. Results: One hundred and thirty one patients with MCTD were followed at our centre. We identified 14 patients with renal involvement with a prevalence of $10.7 \%$. Among those patients, 13 were women $(92.8 \%)$; mean age at onset of renal involvement was $44 \pm 8$ years. Most frequent manifestations were Raynaud's phenomenon in $13(92.8 \%)$ patients, arthritis in 12 (85.7\%), puffy hands in 12 $(85.7 \%)$, sclerodactily in $8(77.1 \%)$, sicca syndrome in $8(77.1 \%)$ and myositis in 7 $(50 \%)$. Median time elapsed from MCTD diagnosis to renal involvement was 83 (2-365) months. In 3 patients, renal involvement was present at MCTD onset. Seven $(50 \%)$ patients had other signs of MCTD activity at the time of renal involvement onset. Four (28.5\%) patients presented with sub- nephrotic proteinuria, 3 $(21.4 \%)$ with nephrotic range proteinuria and kidney injury, $2(14.3 \%)$ with subnephrotic proteinuria and kidney injury, $2(14.3 \%)$ with nephrotic range proteinuria, 1 only with nephrotic range proteinuria and $1(7.1 \%)$ with end-stage renal disease. Microscopic hematuria was present in $9(64.3 \%)$ patients and leukocyturia in 6 $(42.8 \%)$. Renal biopsy was performed in 8 (57\%) patients; pathological diagnoses were: crescentic and necrotizing glomerulonephritis (GN) (2 patients; one of these patients developed positive ANCA antibodies), GN ISN/RPS 2003 class III+V, ${ }^{1}$ GN ISN/RPS 2003 class III+V with thrombotic microangiopathy, ${ }^{1}$ GN ISN/RPS 2003 class IV+V and vasculopathy, ${ }^{1}$ membranous $\mathrm{GN},{ }^{1}$ minimal mesangial $\mathrm{GN}^{1}$ and chronic tubulointerstitial nephritis with vasculopathy. ${ }^{1}$ Ten $(71.4 \%)$ patients achieved either total or partial remission at a median follow up of 82 (1-367) months. Only one patients required dialysis. At last follow up the median SLICC/ ACR-DI was $1.5(0-4)$ points. Two patients died.

Conclusions: In our cohort of MCTD patients, prevalence of renal involvement was low, although higher than the one reported in other populations. Clinical presentation and pathological diagnoses were diverse. Renal biopsy was helpful, since glomerulonephritis, vasculopathy and overlap with ANCA associated vasculitis were found in several patients; these options should be considered in the differential diagnoses of MCTD patients with renal involvement.

Acknowledgements: No acknowledgements to report.

Disclosure of Interest: None declared

DOI: 10.1136/annrheumdis-2018-eular.7455

\section{FRI0441 IDIOPATHIC INFLAMMATORY MYOPATHIES: CLINICAL CHARACTERISTICS, SURVIVIAL AND POOR PROGNOSTIC FACTORS OF 110 PATIENTS FROM TURKEY}

E. Oguz, M. Erdugan, B.A. Esen, A. Gul, L. Ocal, M. Inanc. Department of Internal Medicine, Division of Rheumatology, Istanbul Faculty of Medicine, Istanbul University, Istanbul, Turkey

Objectives: In this study, we aimed to assess clinical features, poor prognostic factors and survival analysis of patients with Idiopathic inflammatory myopathy (IIM).

Methods: Records of 110 patients with IIM that had at least 6 months of follow up, fulfilling Bohan and Peter's criteria were analysed for this study. Survival analysis was done by using Kaplan Meier method and multiple "Cox" regression analysis was applied to calculate the effect of multipl factors.

Results: Sixty-eight percent of 110 patients was female, the mean age of the patients was 46 years, and the average follow up time was 77.5 months. Diagno sis of these patients was dermatomyositis (DM) in $68 \%$, polymyositis (PM) in $26 \%$, autoimmune necrotizing autoimmune myopathy (NOM), inclusion body miyositis (IBM) $6 \%$. The percentage of periungual erythema, arthritis, dysphagia, respiratory muscle involvement and interstitial lung disease(ILD) 56, 22, 24, 32, 11 and 30. Malignancy was identified in $26 \%$ of patients. The percentages of malignan cies diagnosed at the time of diagnosis, before the diagnosis and during the follow-up were 3.6, 11.8 and 8.2. The most frequent malignancy was breast cancer Others are carcinoma of gastrointestinal tract, lung, and genitourinary tract. ANA was present in $36 \%$ and $12 \%$ of patients was positive for anti-Jo- 1 antibody. The average daily dose of prednisolone was $7.5 \mathrm{mg}$, the average usage time was 35.5 months. Causes of death were aspiration pneumonia-sepsis (50\%) and malig nancy (25\%). Significant associations with mortality was found between systemic symptoms, periungual erytema, respiratory muscle involvement, dysphagia, presence of malignancy. Mortality was higher in ANA negative patients $(p<0.001)$ Five and 10 year survival in these patients were $83 \%$ and $75 \%$. Five year survival rate in patients with respiratory muscle involvement was $38 \%$ and $68 \%$ in those with dysphagia. The presence of systemic symptoms, and malignancies were identified as risk factors for mortality in multivariable analysis.

Conclusions: ILD and malignancies are frequent in our IIM cohort. Malignancies are mostly detected at diagnosis. The mortality rate was high and the most common cause was infection. 10 year survival rate was $79 \%$. Malignancy, respiratory muscle involvement, dysphagia, negative ANA have a detrimental effect survival in IIM patients.

Disclosure of Interest: None declared

DOI: 10.1136/annrheumdis-2018-eular.4905

\section{FRI0442 LONG-TERM TREATMENT WITH RITUXIMAB IN INTERSTITIAL LUNG DISEASE RELATED TO SYSTEMIC SCLEROSIS: OUR CLINICAL EXPERIENCE}

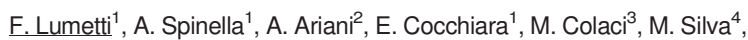
N. Sverzellati ${ }^{4}$, C. Ferri ${ }^{1}$, C. Salvarani ${ }^{1}$, D. Giuggioli ${ }^{1}{ }^{1}$ Scleroderma Unit, Chair of Rheumatology, University of Modena and RE, Modena; ${ }^{2}$ Department of Medicine, Internal Medicine and Rheumatology Unit, Azienda Ospedaliero-Universitaria di Parma, Parma; ${ }^{3}$ Internal Medicine Unit, Cannizzaro Hospital, University of Catania, Catania; ${ }^{4}$ Department of Clinical Sciences, Section of Radiology, University of Parma, Parma, Italy

Background: Systemic sclerosis (SSc) is an immune-mediated disorder charac terised by abnormal fibrosis and diffuse microangiopathy with skin and internal organ involvement. Interstitial lung disease (ILD) represents one of the most challenging complication of SSc, difficult to manage and correlate with a poor prognosis. Chest Computed Tomography (CT) is the gold standard for detection and evaluation of SSc-ILD by means of semi-quantitative scoring of extent of lung involvement. Some preliminary data suggest that rituximab (RTX) may be usefully employed in the treatment of SSc patients.

Objectives: To investigate the role and effect of RTX on ILD in our SSc patients series.

Methods: We retrospectively evaluated a series of $18 \mathrm{SSc}$ patients (M/F 6/12 mean age 54.6 \pm 17.6 SD years, mean disease duration $11.4 \pm 6.5$ SD years, L/D cutaneous subsets 6/12) who received one or more cycles of RTX (4 weekly infusions of $375 \mathrm{mg} / \mathrm{m} 2$ ) every 6 months for a total of $1-6$ cycles. Lung involvement was studied by means of pulmonary function tests (PFTs) (18/18) and inspiratory 
chest CT (10/18) before and after treatment. In particular, we considered PFTs performed 6 months before RTX, at time 0 , one year after the first RTX cycle, and at the end of follow-up (13.7 \pm 7.3 years). ILD extent score was assessed by the semi-quantitative method proposed by Goh et al (2008). ${ }^{1}$

Results: Forced vital capacity (FVC\%) significantly reduced during the year before RTX treatment [from $95.2 \pm 17.4$ to $84.8 \pm 16.4 ; \mathrm{p}=0.0017$ ], as well as the diffusing capacity for carbon monoxide (DLCO\%) [from $58.1 \pm 14.3$ to $47.6 \pm 12.9$; $\mathrm{p}=0.0002]$. Conversely, FVC\% and DLCO $\%$ stabilised one year after the first RTX cycle $(80.8 \pm 23$ and $47.8 \pm 15.7$, respectively), and at the end of the follow-up ( 84.3 \pm 24.6 and $54.8 .8 \pm 12.3$, respectively; $p=0.0001$ ). In our cohort, only $7 / 10$ patients had ILD detectable on CT before treatment. At the end of the follow-up we observed that ILD extented in 6/7 cases one patient remained stable, the three subjects without ILD did not developed pulmonary fibrosis.

Conclusions: According to PFTs results, our study showed that RTX could stabilise the progression of lung function tests in SSc patients. However, the semiquantitative visual score identified radiological pulmonary worsening in many patient with stabilised PFTs. Therefore, the correlations between functional and radiological outcomes are so weak that many Authors suggested they should be considered together in SSc-ILD assessment.

\section{REFERENCE:}

[1] Goh NSL, Desai SR, Veeraraghavan S, et al. Interstitial lung disease in systemic sclerosis: a simple staging system. Am J Respir Crit Care Med 2008;177:1248-54.

Disclosure of Interest: None declared DOI: 10.1136/annrheumdis-2018-eular.6052

\section{FRI0443 DIGITAL ARTERY VOLUME INDEX: THE FIRST OBJECTIVE, AUTOMATED, NON-INVASIVE IMAGING DIAGNOSTIC OF MACROVASCULAR INVOLVEMENT IN SSC}

G. Lettieri ${ }^{1}$, G. Abignano ${ }^{1}$, G. Bagnato ${ }^{1}$, S. Eng ${ }^{1}$, J.P. Ridgeway ${ }^{1}$, J.N. Kaftan ${ }^{2}$, M. Hinton ${ }^{2}$, O. Kubassovaa ${ }^{2}$, M.H. Buch ${ }^{1}$, P. Emery ${ }^{1}$, P. O'Connor ${ }^{1}$, F. Del Galdo ${ }^{1}$. ${ }^{1}$ Leeds Institute of Rheumatic and Musculoskeletal Medicine, University of Leeds, Leeds; ${ }^{2}$ IAG, Image Analysis Group, London, UK

Background: Macrovascular involvement in Systemic Sclerosis (SSc) is caused by proliferation of vascular smooth muscle cells within the intima of arteries (neointimal proliferation). The resulting decrease in arterial volume is responsible for the severity of Raynaud's and for the onset of severe clinical manifestations such as Renal Crisis and Pulmonary Artery Hypertension.

Several RCTs have demonstrated the efficacy of Endothelin Receptor Antagonists (ERAs) in targeting neointimal proliferation, which makes them to date, the only disease modifying agent available in SSc. Nevertheless, the lack of validated early diagnostics means the intention to treat with ERAs remains limited to the diagnosis of end stage clinical manifestations of neointimal proliferation, such as presence of Digital Ulcer (DU) and PAH.

Objectives: Here we aimed to determine the proof of concept validity of automated Digital Artery Volume Index (DAVIX) measured by non-contrast Time of Flight (TOF) MRI as an objective diagnostic tool to be used as surrogate outcome measure of neointimal proliferation in SSc.

Methods: 10 Healthy Volunteers (HV) and 8 SSc patients were enrolled. Six patients underwent longitudinal assessments at least 12 months apart. MRI scans were performed on a 3T Magnetom Verio (Siemens) and consisted of a VIBE 3D T1 scan and a 2D TOF sequence of $8 \mathrm{~min}$. DAVIX was calculated as the percentage of the ratio of digital artery and the respective finger volumes. The vessels and fingers were manually outlined by an expert radiologist and used as a 'gold standard' (DAVIX-1) and compared to a 2nd independent radiologist assessment (DAVIX-2). An automated segmentation algorithm was developed using threshold based segmentation and region growing (DAVIX-A) and validated against gold standard. Intraclass correlation coefficient (ICC) and absolute agreement were calculated to estimate reliability. Bland-Altman bias and $95 \%$ limits of agreement (LoA) were calculated as well.

Results: SSc patients and HV had comparable age (44 \pm 8 vs $45 \pm 8$ ) and gender $(F: M=5: 2$ vs $4: 2$ ). 4 fingers were affected by DUs at baseline and two fingers were affected by new DU at follow up. Mean $( \pm S D)$ DAVIX in HV was $1.21( \pm 0.39)$ with no significant difference among individual fingers. Mean DAVIX in SSc patients was $0.37( \pm 0.18)$ and $0.32( \pm 0.39)$ at baseline and follow-up, respectively $(p<0.0001$ vs HV for both). The fingers with DU had an average DAVIX of 0.24 $( \pm 0.08)$ vs $0.40( \pm 0.18)$ of the fingers without $D U(p=0.02)$. DAVIX changed over time in both directions. In the fingers affected by new DU, DAVIX dropped by $56 \%$ ( 0.161 vs 0.072 ) and $86 \%$ (1.149 vs 0.132$)$, respectively. ICC among two independent readings was $0.90(95 \%$ LoA $-0.139,0.118)$ with overall $r^{2}=0.74$ $(\mathrm{p}<0.0001)$. Automated segmentation showed superior correlation to gold standard with $r^{2}=0.80(p<0.0001)$.
Conclusions: This proof of concept study demonstrated validity and sensitivity to change of DAVIX for the automated volumetric assessment of digital arteries, which reflected clinical worsening in patients with new DU. Larger, longitudinal studies are planned to assess DAVIX' predictive value for the onset of DU and its potential use as early diagnostic of neointimal proliferation in SSc.

Disclosure of Interest: None declared

DOI: 10.1136/annrheumdis-2018-eular.6616

\section{FRI0444 PERIOSTIN IN SYSTEMIC SCLEROSIS: SERUM LEVELS AND SKIN EXPRESSION OF A NOVEL POSSIBLE BIOMARKER}

G. De Luca ${ }^{1}$, C. Campochiaro ${ }^{1}$, S. Franchini ${ }^{1}$, S. Burastero ${ }^{2}$, S. Sartorelli ${ }^{1}$, A. Giachi' ${ }^{1}$, G. Cavalli ${ }^{1}$, C. Doglioni ${ }^{3}$, L. Dagna'. ${ }^{1}$ Unit of Rheumatology, Immunology, Allergy and Rare Diseases (UnIRAR); ${ }^{2}$ Cellular and molecular allergy; ${ }^{3}$ Unit of Pathology, San Raffaele Scientific Institute, Milan, Italy

Background: Periostin(PN), a matricellular protein, serves as a regulator of wound healing and fibrosis. $\mathrm{PN}^{-/-}$mice develop lower degrees of fibrosis when exposed to bleomycin(BLM), suggesting a possible role in the pathogenesis of Systemic Sclerosis(SSc) ${ }^{1}$. PN serum levels are increased in SSc and seem to be associated with skin disease severity ${ }^{2}$

Objectives: To evaluate the role of serum PN as a biomarker of SSc severity, and to determine PN tissue expression in SSc patients.

Methods: PN serum levels were assessed by ELISA in 48 patients: 3 primary Raynaud's, 12 early SSc, 11 SSc without organ involvement, 22 SSc with organ involvement. All SSc patients met 2013 ACR/EULAR criteria. PN serum levels were evaluated in 28 sex-/age-matched healthy-controls(HCs). Data regarding disease subtypes and organ involvement were correlated. PN skin expression was determined by immunohistochemistry on paired involved and uninvolved skin biopsy samples in 10 patients ( 4 lcSSc and 6 dcSSc) in combination with a-SMA CD68, CD3, CD4, CD8, CD163, CD20 and CD131.

Results: PN serum levels were higher in SSc patients compared to $\mathrm{HCs}(32.7$ \pm 8.0 vs $27.7 \pm 7.3 \mathrm{ng} / \mathrm{ml}, \mathrm{p}<0.001$ ). Its levels were comparable among different groups. No differences in PN serum levels were detected when comparing disease subtypes, disease duration, presence and extent of organ involvement, autoantibodies profile and current or previous treatment. Higher PN levels were found in SSc patients with active pattern at nailfold videocapillaroscopy and a his tory of digital ulcers $(p<0.02)$. PN serum levels did not correlate with skin or lung disease extent. Skin samples from involved SSc skin showed high PN expression in the upper dermis and in the fibrotic area of the lower dermis(more evident in dcSSc), suggesting a role in skin fibrosis. In all SSc involved skin, PN was expressed in areas where ongoing fibroproliferation and macrophage/T lymphocytic infiltration occured, indirectly suggesting a pathogenic role in inflammation driven-fibrosis. Interestingly, an identical PN immunohistochemical expression was evident in uninvolved dcSSc skin, but not in lcSSc skin

Conclusions: In our cohort PN serum levels are elevated in SSc patients but they do not correlate with disease features. Its postulated role as a severity biomarker needs to be further elucidated. The different immunohistochemical expression of PN in uninvolved skin from dcSSc and IcSSc patients suggests a possible pathogenic role in the progressive inflammation-driven fibrosis that characterise diffuse cutaneous involvement.

\section{REFERENCES}

[1] Yang L, et al. Periostin facilitates skin sclerosis via PI3K/Akt dependent mechanism in a mouse model of scleroderma. PLoSOne 2012

[2] Yamaguchi $Y$, et al. Serum Periostin levels are correlated with progressive skin sclerosis in patients with systemic sclerosis. Br J Dermatol 2013.

Disclosure of Interest: None declared

DOI: 10.1136/annrheumdis-2018-eular.5975

\section{FRI0445 A RANDOMISED CONTROLLED TRIAL TO COMPARE THE EFFICACY OF ORAL MYCOPHENOLATE MOFETIL WITH PLACEBO IN PATIENTS WITH SYSTEMIC SCLEROSIS RELATED EARLY INTERSTITIAL LUNG DISEASE}

G. Naidu ${ }^{1}$, S. Sharma ${ }^{1}$, V. Dhir ${ }^{1}$, S. Dhooria ${ }^{2}$, A. Sinha ${ }^{3}$, A. Mb ${ }^{1}$, S. Jain ${ }^{1} .{ }^{1}$ Internal Medicine; ${ }^{2}$ Pulmonary Medicine; ${ }^{3}$ Radiodiagnosis, PGIMER, Chandigarh, India

Background: Previous studies showed benefit of immunosuppressants in moderate to severe ILD in systemic sclerosis (SSc). ${ }^{1,2}$ Initiation of immunosuppression early in the course of SSc-ILD might help in halting disease process and improve long term morbidity and mortality.

Objectives: Aim of the study was to determine efficacy and safety of mycophenolate mofetil (MMF) in treating early and mild SSc-ILD (ILD on HRCT, FVC $\geq 70 \%$ 